Submitted to the Astrophysical Journal

\title{
Ultra High Energy Cosmic Rays and the Large Scale Structure of the Galactic Magnetic Field
}

\author{
Todor Stanev \\ Bartol Research Institute, University of Delaware, Newark DE 19711, U.S.A.
}

\begin{abstract}
We study the deflection of ultra high energy cosmic ray protons in different models of the regular galactic magnetic field. Such particles have gyroradii well in excess of 1 kpc and their propagation in the Galaxy is not affected by the small scale structure of the field. Their trajectories, however, reflect the large scale structure. A future large experimental statistics of cosmic rays of energy above $10^{19} \mathrm{eV}$ could be used for a study of the large scale structure of the galactic magnetic field if such cosmic rays are indeed charged nuclei accelerated at powerful astrophysical objects and if the distribution of their sources is not fully isotropic.

We also show the corrections of the arrival directions due to the deflection in the galactic magnetic field of for a subsample of the world statistics of cosmic rays of energy above $2 \times 10^{19} \mathrm{eV}$ and discuss the implications for the search for the sources of these events.
\end{abstract}

Subject headings: magnetic fields - structure - Galaxy: cosmic rays - magnetic fields Galaxy

\section{Introduction}

While the majority of cosmic rays detected at Earth is quite isotropic, an observable anisotropy appear to set up (Watson 1991) at the approach of energies of order $10^{18} \mathrm{eV}(1 \mathrm{EeV})$. This effect is quite natural, because the gyroradius of protons of that energy in $1 \mu \mathrm{G}$ magnetic field is about $1 \mathrm{kpc}$, i.e. of $\mathrm{O}(10)$ larger than the scale of the random component of the galactic magnetic field (GMF). Although the typical values of the regular component of the GMF are expected to be 4-6 times higher for most of our Galaxy (Beck et al. 1996), the cosmic ray spectrum extends to energies higher by at least 2 and a half orders of magnitude. This creates an opportunity to study the large scale structure of GMF through the observations of the arrival directions of the ultra high energy cosmic rays (UHECR), those of energy above $10^{19} \mathrm{eV}$.

UHECR are observed by the air showers that they initiate when the primary UHECR particles interacts in the atmosphere. Ground based air shower arrays register the arrival of a large shower 
by the coincidental arrival of a large number of charged particles in distant particle detectors. Since the flux of UHECR is extremely low ( 0.5 per $\mathrm{km}^{2}$ per year per steradian above $10^{19} \mathrm{eV}$ ) the shower arrays designed for their detection are by necessity very sparse and the detection yields only the energy and the arrival direction of the primary UHECR. The nature of that particle could be only derived from a large enough statistical sample.

Although the origin, and even the nature, of the cosmic rays with such high energy is yet unknown, it is easy to understand their value for revealing the general structure of GMF in the natural assumption that they are mostly ionized hydrogen atoms (protons) and are of extragalactic origin. Protons of energy above $10^{19} \mathrm{eV}$ do not suffer significant energy loss on galactic scalelengths. They are only deflected in magnetic fields extending on scale larger than $1 \mathrm{kpc}$. If their arrival distribution on their entry into our Galaxy is not strictly isotropic, GMF would have a focusing (or defocusing) effect on their arrival distribution at Earth, which will reflect the general GMF structure, rather then the local magnetic field in the vicinity of the solar system. This is of course only possible if the UHECR are nuclei of astrophysical origin (Hillas 1984, Szabelski et al 1986, Rachen and Biermann 1993), rather than gamma rays generated in exotic processes, such as topological defects (Bhattacharjee et al. 1992, Gill and Kibble, 1994).

The current experimental statistics of such events is not sufficiently large to make definite conclusions on their arrival directions, although some potentially significant anisotropies have been recently observed by two independent groups (Stanev et al. 1995, Hayashida et al. 1996). The Auger project (The Auger Collaboration 1995), however, proposes building of two identical air shower arrays, situated in the Northern and in the Southern hemispheres in such a way that they provide all sky coverage. In few years of observation these detectors would increase the world UHECR statistics by more than a factor of 10 .

As we will show further down, such much improved experimental statistics will contribute not only to the important question of the origin of the UHECR, but also to our knowledge of the large scale structure of the galactic magnetic field. This article is organized in the following way: in section 2 we introduce the galactic magnetic field models used in this study. Section 3 gives the method of proton propagation in GMF models and the general results. Section 4 shows how the arrival directions of an experimental sample of UHECR is affected by the chosen GMF models and gives the general conclusions from this study.

\section{The galactic magnetic field models.}

We have build two galactic magnetic field models, which implement the general ideas of the large scale structure of the galactic magnetic field and incorporate the knowledge stemming from experimental observations. Observations of our Galaxy, as well of many other galaxies (Beck et al. 1996, Kronberg 1994), show that the regular component of the galactic magnetic fields could be well described by spiral fields with $2 \pi$ (axisymmetric, ASS) or $\pi$ (bisymmetric, BSS) symmetry. 
In $z$-direction, i.e. perpendicular the the galactic plane, the fields are either of odd (dipole type) or even (quadrupole type) parity, A and S type respectively (Beck et al. 1996). There are also observations of magnetic fields of mixed type.

As we show further down, these four general types of magnetic field structure would affect the propagation of UHECR in different ways. We have therefore made two extreme combinations and build 1). a bisymmetric field model with field reversals and odd parity (BSS_A model); and 2). an axisymmetric field model without field reversals and with even parity (ASS_S model). Both models incorporate the following observational knowledge:

1 ). The solar system is at a galactocentric distance $R=8.5 \mathrm{kpc}$. The local magnetic field in the vicinity of the solar system has a strength of $\sim 2 \mu \mathrm{G}$ in direction of $l=90^{\circ}$. The GMF pitch angle $p$ was taken to be $-10^{\circ}$ (Rand and Lyne 1994).

2 ). There is a reversal of the magnetic field (in the BSS_A model) at $l=0^{\circ}$ at distance of 0.5 $\mathrm{kpc}$ and a second reversal at distance of $\sim 3 \mathrm{kpc}$ (Rand and Lyne 1994, Vallée 1991). In the ASS_S model there are no reversals and the magnetic field strength decreases to zero at the same locations.

The field strength at a point $(r, \theta)$ in the galactic plane is

$$
B(r, \theta)=B_{0}(r) \cos \left(\theta-\beta \ln \frac{r}{r_{0}}\right),
$$

for the bisymmetric model, where $r_{0}$ is the galactocentric distance of the location with maximum field strength at $l=0^{\circ}$ and $\beta=1 / \tan p=-5.67$. In our representation $r_{\circ}=10.55 \mathrm{kpc}$. The BSS_A model is very much like the model of (Han and Qiao, 1994) scaled to a galactocentric distance of $8.5 \mathrm{kpc}$. In the ASS_S model

$$
B(r, \theta)=B_{0}(r)\left|\cos \left(\theta-\beta \ln \frac{r}{r_{0}}\right)\right|,
$$

The $\theta$ and $r$ components of the field are correspondingly

$$
B_{\theta}=B(r, \theta) \cos p, \quad B_{r}=B(r, \theta) \sin p .
$$

There is no $z$ component in the basic models. $B_{0}(r)$ is taken to be $3 R / r \mu \mathrm{G}$ as in (Sofue and Fujimoto 1983), i.e. $6.4 \mu \mathrm{G}$ at $\mathrm{r}=4 \mathrm{kpc}$ and constant at that value in the central region of the Galaxy. This radial dependence is consistent with the field strengths inferred from pulsar rotation measures (Rand and Lyne 1994). In our models the field extends to galactocentric distances of 20 $\mathrm{kpc}$ in all directions.

The size and field strength in the galactic halo is extremely important for the proton trajectories. We have assumed an extended halo with two scaleheights. The field strengths above and below the galactic plane are

$$
|B(r, \theta, z)|=|B(r, \theta)| \exp \left(z / z_{0}\right)
$$


with $z_{0}=1 \mathrm{kpc}$ for $|z|<0.5 \mathrm{kpc}$ and $z_{0}=4 \mathrm{kpc}$ for $|z|>0.5 \mathrm{kpc}$. The odd parity (BSS_A) model preserves the field direction at the disk crossing while the even parity (ASS_S) model changes it.

Fig. 1 shows the field strength and direction in the galactic plane for both models.

\section{Proton propagation in the galactic magnetic field}

To follow the particle trajectory in the GMF we use a well known technique which is standard in determining the geomagnetic cutoffs for low energy cosmic rays and their asymptotic directions - backtracking antiprotons (Flückiger et al. 1991, Bieber et al. 1992) injected at Earth in different directions. The negatively charged antiprotons injected in certain direction at Earth will follow exactly the same trajectory as a positively charged proton arriving at Earth from the same direction. So we inject antiprotons at different galactic longitude $b$ and latitude $l$ at the location of the solar system and follow their propagation in the GMF models until they reach a distance of $20 \mathrm{kpc}$ from the galactic center. Then we calculate the 'true' values of $(l, b), l_{\text {true }}$ and $b_{\text {true }}$, from which the particle reached the Galaxy before its direction was changed by the GMF. We backtracked the antiprotons by integrating the equations of motion of charged particles using an Runge-Kutta method with adaptive stepsize control (Lipari and Stanev 1995).

Fig. 2 shows the results for the two GMF models. Protons of energy 100, 80, 60, 40, and 20 $\mathrm{EeV}$ were injected at Earth at galactic latitudes from $b=-75^{\circ}$ to $b=+75^{\circ}$ and longitudes from $l=45^{\circ}$ to $l=315^{\circ}$. The deflection for a total of 304 injection directions were calculated. The region of the galactic center was intentionally omitted from the calculation because of the very large uncertainty in the GMF magnitude and structure. Each of the shown trajectories shows the deflection suffered by a proton as a function of its energy, as indicated on the legend to Fig. 2. The origin of each trajectory is the injection direction, and the arrow ends in the direction at which a $20 \mathrm{EeV}$ proton would arrive at the galactic boundary (20 kpc from the galactic center) to be observed as coming from the injection direction at Earth.

Let us first examine the proton trajectories for model BSS_A. There is a general trend for a flow from North to South, which is quite strong in the direction of the galactic anticenter. There is also a general trend for a flow in the direction away from the galactic center for longitudes $b$ less than about $-20^{\circ}$, and a flow towards the galactic center at the corresponding Northern longitudes. One has to be reminded that arrows in Fig. 2 show the reverse trajectory - a particle arriving at the Galaxy from the position of the tip of the arrow would be observed at Earth at its origin. The RMS deflection angles $\left(\Delta \Psi_{R M S}\right)$ are shown in Table 1 as a function of the proton energy. As could be seen from Fig. 2 deflection angles are a strong function of the injection direction. The variations in the magnitude of the deflection angle are large and the average deflection angle $\langle\Delta \Psi\rangle$ is always smaller than the RMS value. $\Delta \Psi_{R M S}$ values could only be taken as a guide for the magnitude of the expected deflection and its energy dependence, which is slightly stronger than linear for energies below $40 \mathrm{EeV}$. 
Fig. 2b (model ASS_S) shows quite a different picture. To start with, the deflections are quite a bit stronger. The main reason is that in the axisymmetric model protons always propagate through magnetic field of the same polarity (except in the equatorial region), while in the bisymmetric model they move from regions of positive polarity into regions of negative polarity and vice versa where they are respectively deflected in opposite directions. Because of this the net deflection in a bisymmetric field is smaller. The most crucial difference is caused by the even parity of the ASS_S model - there is a very strong flow of protons towards the galactic plane, especially at longitudes between $90^{\circ}$ and $270^{\circ}$. This would make extragalactic cosmic rays appear as if they are actually arriving from the direction of the galactic plane. At high positive and negative longitudes there is now a flow away from $l=0^{\circ}$.

The very large deflections at extreme Northern and Southern longitudes may not be realistic because of the possibly exaggerated size and strength of the galactic halo in our magnetic field models. These are still appropriate for this exercise which is used here to demonstrate the effect of the galactic magnetic field on the particle propagation in the Galaxy.

A possible $z$-component of the galactic magnetic field, that would correspond to the existence of galactic wind, would also have extreme effects on the particle deflection, as shown in Fig. 3, which follows the propagation of protons in the two field models which now have a constant $B_{z}$ component of $0.3 \mu \mathrm{G}$. In the BSS_A model $B_{z}$ is always directed to North, while in the ASS_S model $B_{z}$ points at North in the Northern galactic hemisphere and changes direction at the crossing of the galactic plane.

The existence of a $B_{z}$ component makes the deflection pattern much more complicated, especially in the ASS_S model. Particles appear to change their deflection from positive to negative as a function of their rigidity. These could be recognized as trajectories in Fig. 3 that cross the equatorial plane. The asymmetry in the deflection patterns becomes stronger, and protons injected at very high longitudes are affected the most. Fig. 3 demonstrates how a relatively small, hardly observable, $B_{z}$ component could change the deflection pattern for UHECR in the Galaxy. A more realistic $B_{z}$ component, related to the field strength and direction in the galactic plane, would complicate the deflection pattern even more.

These complications, however, concern mostly the details of the proton propagation in the Galaxy. The main features observable in Fig. 2 are still present in the case of non-zero $B_{z}$ component. The general flows toward the galactic plane (BSS_A) and towards and away from $l=$ $0^{\circ}$ are somewhat modified, but still the major propagation effect.

\section{Discussion and conclusions}

The magnitude of the deflections in the galactic magnetic field requires that the arrival direction of UHECR are corrected for deflection in GMF before their arrival directions are

compared to locations of powerful astrophysical sources. As an example we perform this correction 
in Fig. 4 for the experimental sample that was used in (Stanev et al. 1995) in search of correlations with the the galactic and the supergalactic de Vaucouleurs 1956, de Vaucouleurs 1976) planes. These events come from four air shower arrays that have operated in the Northern hemisphere at different times since 1960's: Volcano Ranch (Linsley 1963, Laurence et al. 1991, Egorov 1993, Yoshida et al. 1995). The total number of events above $2 \times 10^{19} \mathrm{eV}$ is 143 .

It is interesting to observe that the correction in either of the GMF models did not change appreciably the RMS distance of the UHECR direction with respect to the supergalactic plane $b_{R M S}^{S G}$. The RMS distance to the galactic plane in the ASS_S model was increased by several degrees from its original (already unlikely high) value for UHECR of energy above $4 \times 10^{19} \mathrm{eV}$. The distribution about the two planes, however, is now quite a bit different. A closer examination of Fig. 4 reveals several interesting phenomena, that are not strong enough to draw significant conclusions, but are still interesting. The effects are stronger in Fig. 4b, which corresponds to the ASS_S model.

There are several groups of experimental events that seem to be significantly closer to the supergalactic plane after the correction for the deviation in the GMF. One such group could be observed at northern longitudes $\left(b>60^{\circ}\right)$ at $l=180^{\circ}$ to $200^{\circ}$. The correction made all these showers appear as coming from the Virgo cluster. Another group of event that move closer to the supergalactic plane are positioned at $b=0^{\circ}$ to $30^{\circ}$ and $l=140^{\circ}$ to $160^{\circ}$. Those two groups of UHECR could be observed in both models. Quite an interesting development appears in a wide range south of the galactic plane ( $b$ from $-5^{\circ}$ to $-50^{\circ}$ ) at $l=135^{\circ}$ to $160^{\circ}$. Many UHECR in this region land almost exactly on the supergalactic plane after the correction for deflection in the ASS_S GMF model. The effect does not exist in the BSS_A model where the correction takes the UHECR directions to the North.

Events that belong to any of these three groups appear more correlated with the supergalactic plane than before the deflection correction. On the other hand, there is a vast region of the sky $\left(b>0^{\circ}, l<130^{\circ}\right.$, where the corrected UHECR positions are further away from the supergalactic plane. This is the most general trend which can be observed in Fig. 4, which is also essential for net zero result of the correction on $b_{R M S}^{S G}$. The correction also seems to have produced a focusing effect for several UHECR in the region $b$ from $5^{\circ}$ to $15^{\circ}, l$ from $140^{\circ}$ to $160^{\circ}$, which now come together at angles comparable to the angular resolution of the air shower arrays.

Fig. 4 is introduced here only as an example for the propagation effects in the Galaxy. Although the corrected arrival direction maps should be inspected for association with powerful astrophysical objects, one cannot expect to draw any major conclusions on the basis of this statistically limited sample in view of the uncertainties in the galactic magnetic field models. The total world statistics would quickly increase by factors of 10 to 100 when the Auger project comes into operation. Auger proposes two giant air shower arrays with area of $3000 \mathrm{~km}^{2}$ each in both hemisphere. For comparison the biggest operational air shower array (AGASA) has an effective area of $200 \mathrm{~km}^{2}$. After few years of operation, Auger will provide an UHECR statistics of tens 
of thousands. At this stage one could, and should, study the deflections of charged particles in the galactic magnetic field with the dual purpose of establishing the UHECR origin and the large scale structure of the magnetic field.

The magnetic field studies would be most fruitful if we are lucky enough to see individual sources of charged UHECR. Then we would be able to see the highest energy particles coming directly from the source with minimal deflection and lower energy ones creating a halo around them. Such a picture, although not with high enough statistical significance, is suggested by the study of (Stanev et al. 1995). With much higher statistics one should be able to follow the deviation in the GMF as a function of the UHECR energy and derive much more accurate information of the magnetic field structure.

Even now, however, the research presented here helps to understand some previously observed phenomena. In (Stanev et al. 1995) a sudden and strong decrease of the average distance of the UHECR directions to the supergalactic plane was found between energies of 20 and $40 \mathrm{EeV}$. A brief inspection of Table 1 indicates that this is the energy range where the deflection in the galactic magnetic field becomes comparable to the thickness of the supergalactic plane, and that one would expect a strong decrease of any correlation at energy of $20 \mathrm{EeV}$. The average deflection angles also indicate the scale of clustering of UHECR around their potential sources. Another practical application of the presented technique is an analysis of the apparent clustering of the UHECR events detected by the Souther hemisphere SUGAR air shower array (Kewley et al. 1966).

The strength and aspect of the UHECR deflection depends strongly on the viewing area of the particular experiment, which are usually of order 1 steradian. It is thus very interesting to see if the observed shift between the arrival directions of the Haverah Park (Stanev et al. 1995) and AGASA (Hayashida et al. 1996) events in respect with the supergalactic plane could be explained by the different viewing areas of the two experiments, which are at latitudes of $54^{\circ} \mathrm{N}$ and $35.5^{\circ} \mathrm{N}$ respectively. The shift could also be enhanced by a difference in the energy normalization of the two experiments.

The current research does not take into account the possible deflection of charged UHECR in the extragalactic magnetic fields. This would complicate the picture, since the extragalactic fields could be strong enough to cause even bigger deflections on the Mpc extragalactic scalelengths. Kronberg (1994) already suggested to use future UHECR statistics for studies of the extragalactic magnetic fields. The same procedure as the one applied here could be used, at least for some regions of the universe, where the information about the extragalactic magnetic fields is relatively rich, such as the Virgo cluster (Vallée 1993). In any case we have to account for the propagation in our own Galaxy before we go any further.

The author is grateful to R. Beck, P.L. Biermann, R.J. Protheroe J.P. Rachen, D. Seckel and Z.P. Zank for helpful discussions and to M. Nagano and the AGASA group for sharing results 
prior to publication. This work is supported in part by the U.S. Department of Energy under contract DE-FG-91ER40626.

\section{REFERENCES}

The Auger collaboration, 1995, Design Report.

Beck, R., Brandenburg, A., Moss, D., Shukurov, A. and Sokoloff, D., 1996, ARA\&A, in press.

Bieber, J.W., Evenson, P.A. \& Lin, Z., 1992, Antarctic J., 27, 318.

Bhattacharjee, P., Hill, C.T. \& Schramm, D.N., 1992, Phys. Rev. Lett., 69, 567.

Egorov, T.A., 1993, Tokyo Workshop for the Study of Extremely High Energy Cosmic Rays, M. Nagano, ICRC, Tokyo, 35.

de Vaucouleurs, G., 1956, Vistas in Astronomy 2, 1584.

de Vaucouleurs, G., 1976, Second reference Catalog of Bright Galaxies ,Univ. of Texas Press, Austin.

Flückiger, E.O. , Kobel, E., Smart, D.F. \& Shea M.A., 1991, Proc. 22nd Int. Cosmic Ray Conf. (Dublin), 3, 648.

Gill, A.J. \& Kibble, T.W.B., 1994, Phys. Rev. D, 50, 3660.

Han, J.L. \& Qiao, G.J., 1994, A\&A, 288, 759.

Hayashida N., Honda, K., Honda, M., et al., 1996, Phys. Rev. Lett., in press.

Hillas, A.M., 1984, ARA\&A, 22, 425.

Kewley, L.J., Clay, R.W. \& Dawson, B,R., 1996, Astropart. Phys., 5, 69.

Kronberg, P., 1994, Rep. Prog. Phys. 57, 325.

Lawrence, M.A., Reid, R.J.O. \& Watson, A.A., 1991, J. Phys G: Nucl. Part. Phys., 17, 733.

Linsley, J., 1963, Proc. Int. Cosmic Ray Conf. (Jaipur) 4, 77.

Lipari, P. \& Stanev, T., 1995, Proc. Int. Cosmic Ray Conf. (Rome) 1, 516.

Rachen, J.P. \& Biermann, P.L., 1993, A\&A, 272, 161.

Rand, R.J. \& Lyne, A.G., 1994, MNRAS, 268, 497.

Sofue, Y. \& Fujimoto, M., 1983, ApJ, 265, 722. 
Stanev, T. et al., 1995, Phys. Rev. Lett., 75, 3056.

Szabelski, J., Wdowczyk, J. \& Wolfendale, A.W., 1986, J. Phys. G: Nucl. Phys. 12, 1443.

Vallée, J.P., 1991, ApJ, 366, 450.

Vallée, J.P., 1993, MNRAS, 264, 665.

Watson, A.A., 1991, Particle Acceleration in Cosmic Plasmas, Gaisser, T.K. \& Zank, G.P., AIP Conf. Proc. 264, 386.

Yoshida, S., Hayashida, N., Honda, K., et al., 1995, Astropart. Phys., 3, 105.

This preprint was prepared with the AAS LATEX macros v4.0.

Table 1: RMS deflection angle $\left(\Delta \Psi_{R M S}\right)$ as a function of the proton energy in EeV for the models presented in Figs. $2 \& 3$.

\begin{tabular}{l|ccccc}
\hline E, EeV & 100 & 80 & 60 & 40 & 20 \\
& \multicolumn{5}{|c}{$\Delta \Psi_{R M S}$} \\
\hline BSS_A & 3.1 & 3.9 & 5.2 & 7.9 & 17.7 \\
ASS_S & 4.0 & 5.1 & 6.8 & 10.5 & 23.7 \\
ASS_S_Z & 4.5 & 5.7 & 7.6 & 11.8 & 25.9 \\
\hline
\end{tabular}



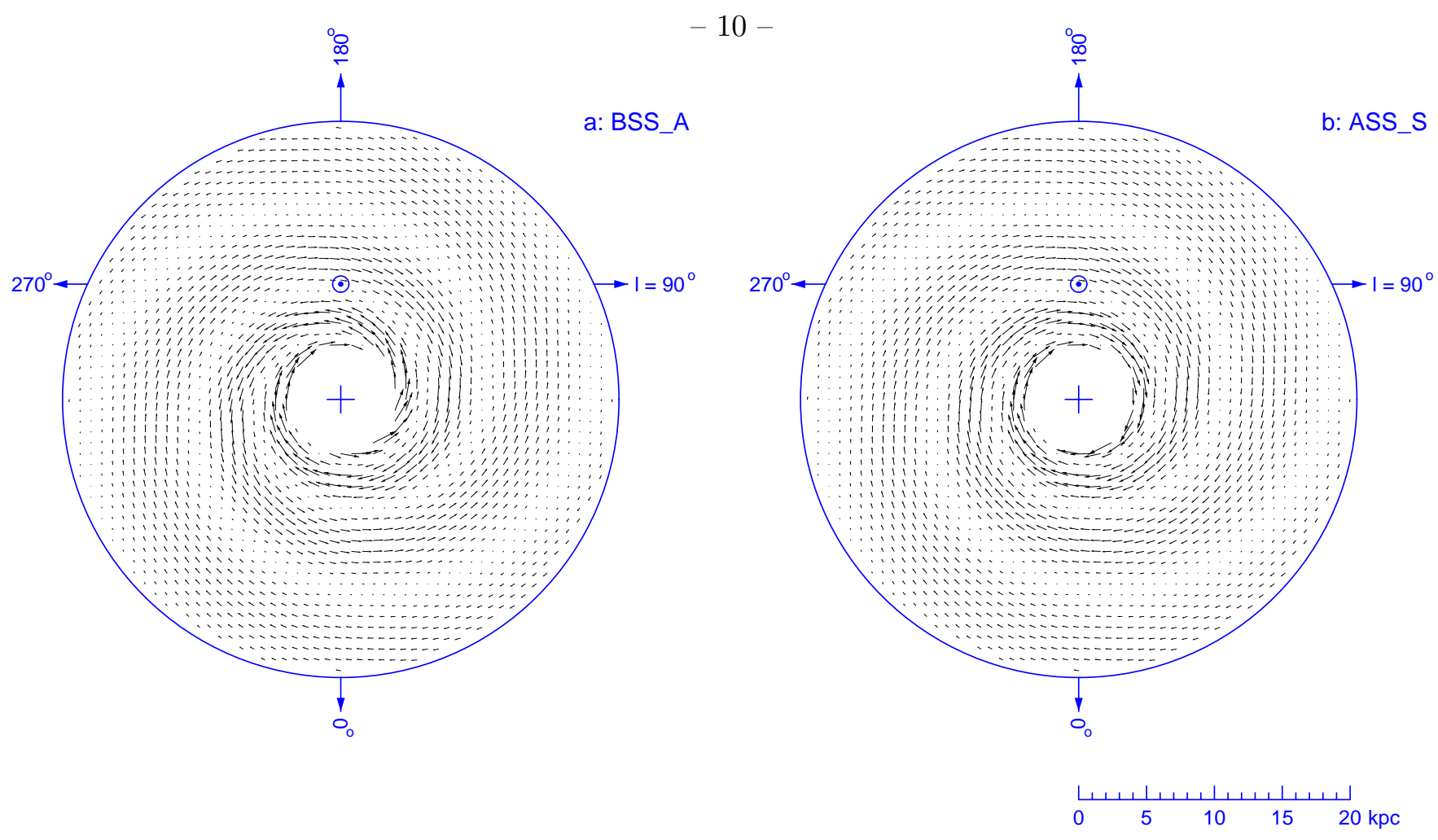

Fig. 1. - The direction and strength of the regular magnetic field in the galactic plane is represented by the length and direction of the arrows for the bisymmetric odd parity model BSS_A (a) and the axisymmetric model with even parity ASS_S (b). The field inside the galactocentric circle of radius $4 \mathrm{kpc}$ follows the general structure of the models with $B_{0}(r)=B_{0}(4 \mathrm{kpc})$. 


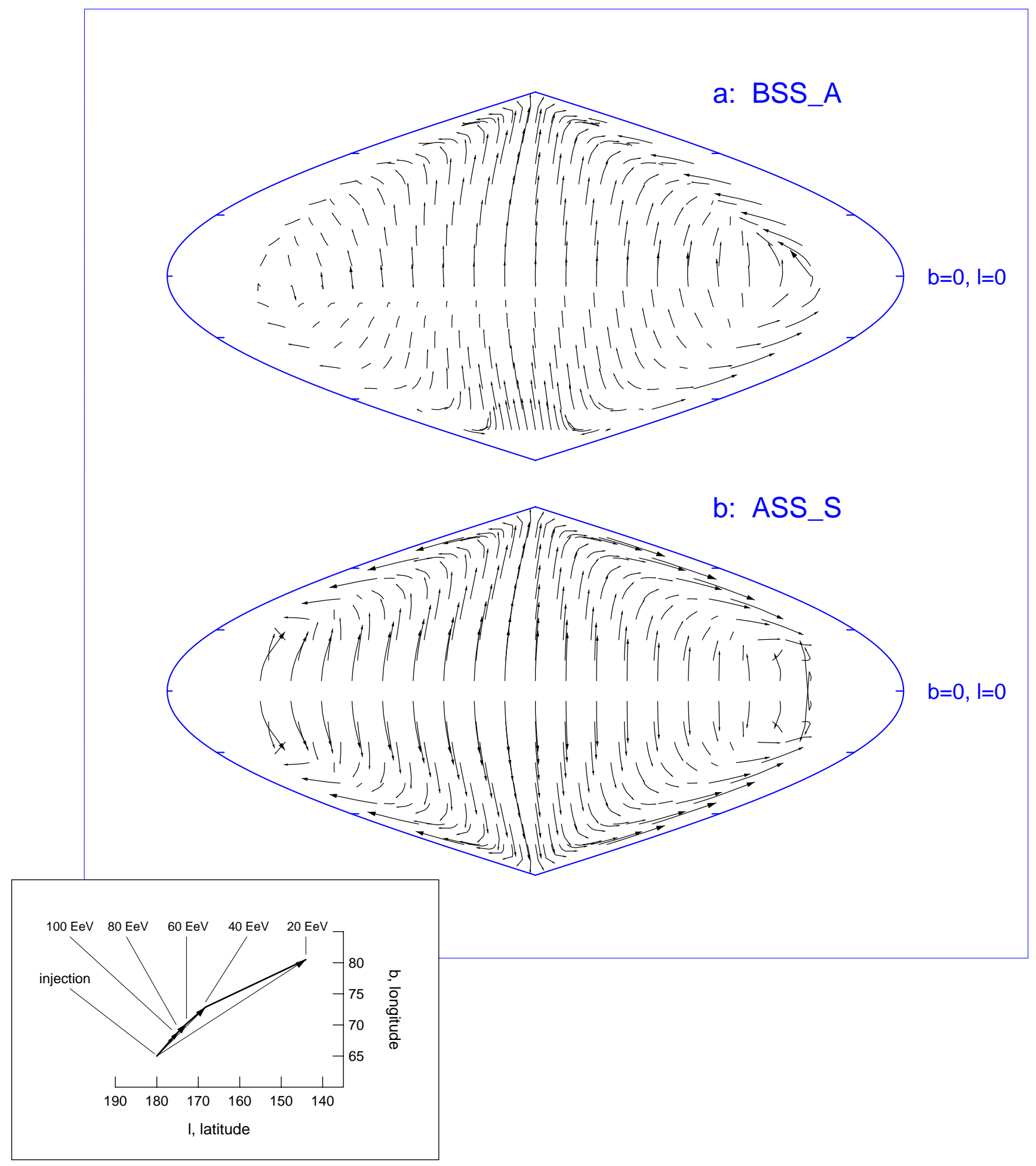

Fig. 2.- Deflection of protons of energy 20, 40, 60, 80, and $100 \mathrm{EeV}$ in the galactic magnetic field for the BSS_A (a) and ASS_S (b) models. The legend in the lower left corner explains how each trajectory is plotted. The particle is injected at Earth in the position of the origin of the plotted trajectory. A proton of energy $100 \mathrm{EeV}$ would move to the angle of the first arrow in its propagation through the Galaxy, a proton of energy $80 \mathrm{EeV}$ would move to the angle of the next arrow, etc. while a proton of energy $20 \mathrm{EeV}$ would move to the tip of the arrow in the main figure. The figure is centered on the galactic anticenter. 


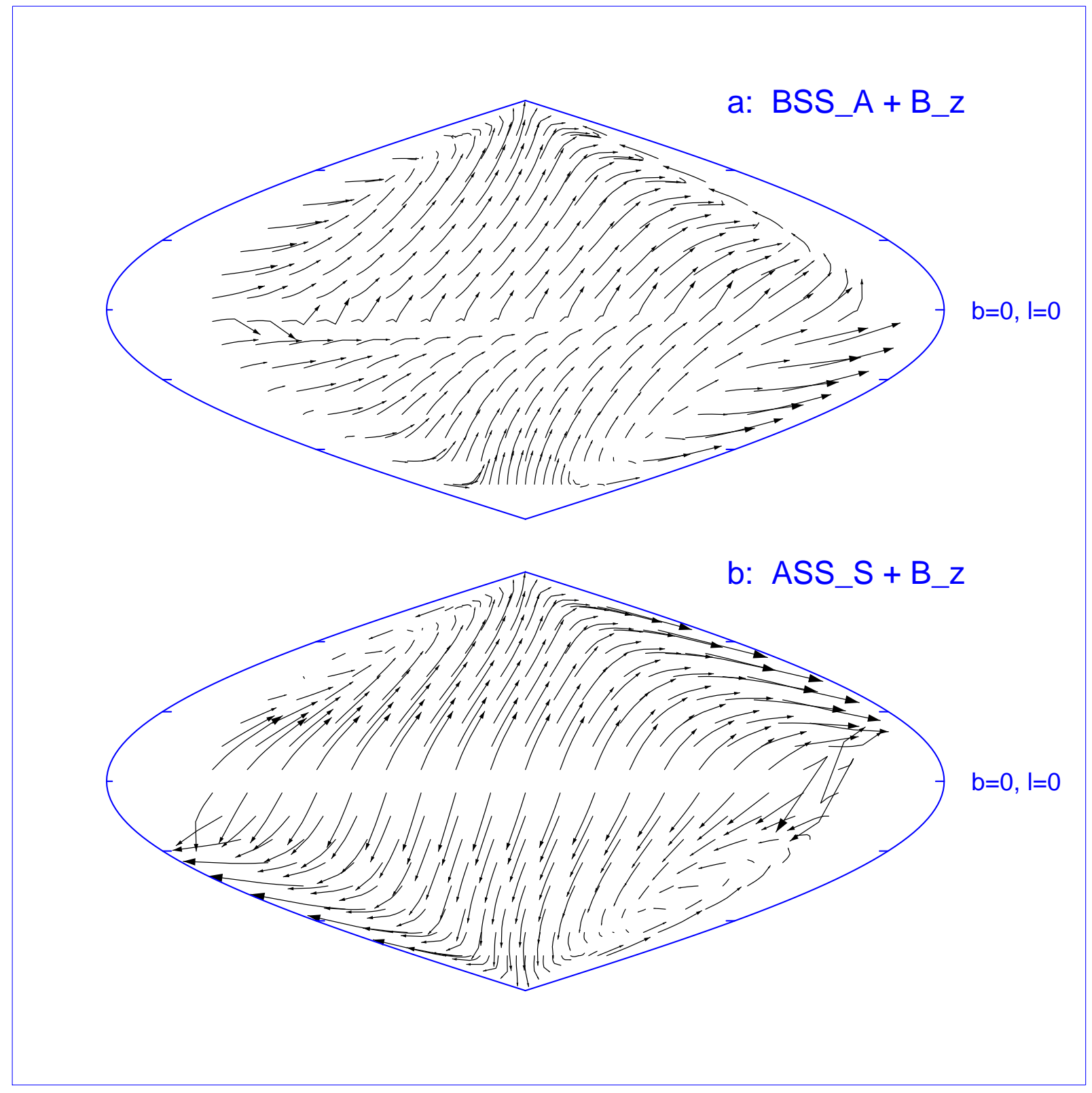

Fig. 3. - Same as Fig. 2 for models with a constant $B_{z}$ component with strength of $0.3 \mu \mathrm{G}$. In the BSS_A case $B_{z}$ always points North, while in the ASS_S case it changes direction at the crossing of the galactic plane. 


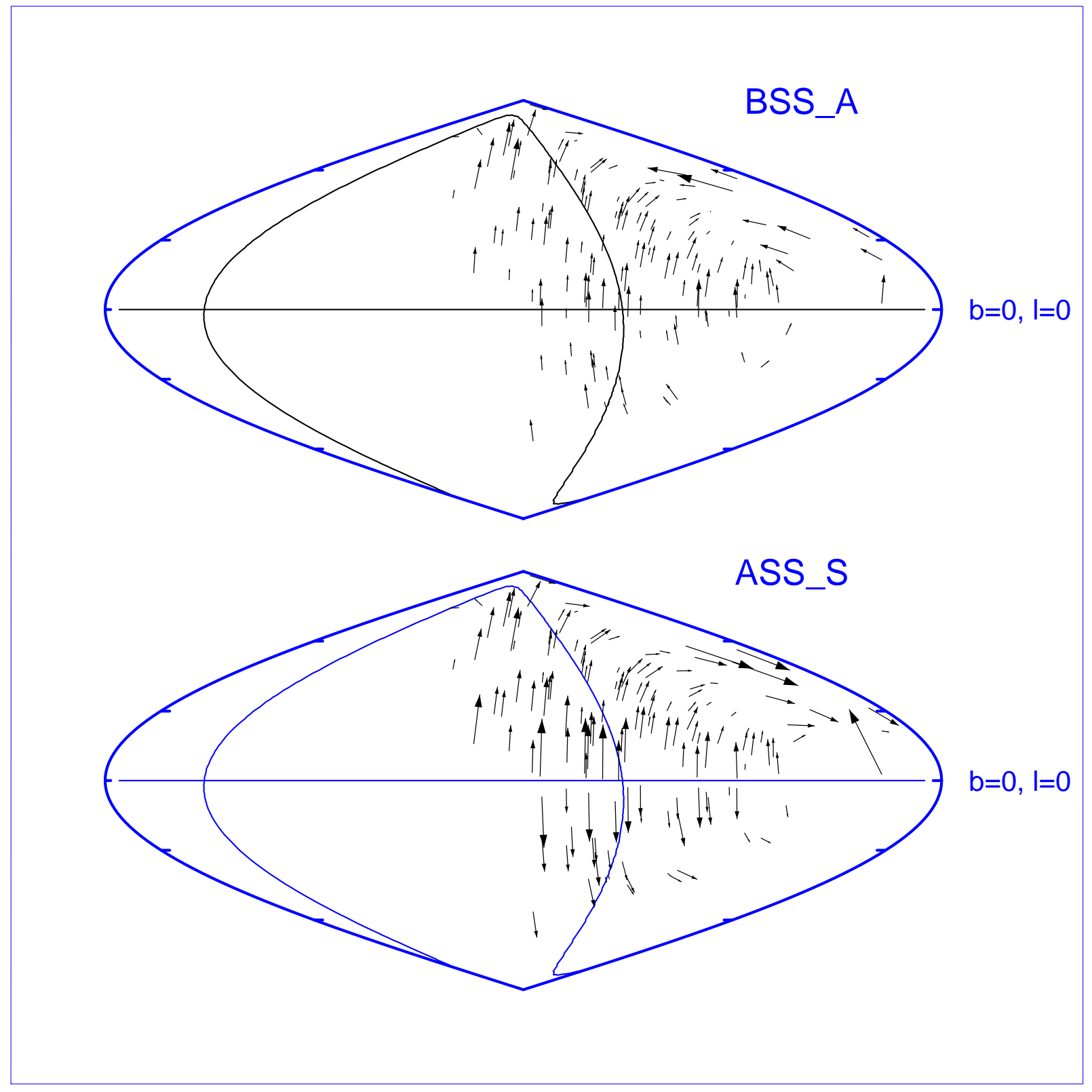

Fig. 4.- Corrections for the arrival directions of the experimental sample used by Stanev et al (1995) in the two magnetic field models. Every experimental event was tracked back from its detection position (origin of trajectory shown) to determine its direction at its arrival in our Galaxy (tip of arrow). 